\title{
Envelope of Family of Angled Projectiles and Its Universal Geometric Characteristics
}

\author{
Haiduke Sarafian \\ The Pennsylvania State University, University College, York, USA \\ Email: has2@psu.edu
}

How to cite this paper: Sarafian, H. (2020) Envelope of Family of Angled Projectiles and Its Universal Geometric Characteristics. American Journal of Computational Mathematics, 10, 425-430. https://doi.org/10.4236/ajcm.2020.103023

Received: June 28, 2020

Accepted: September 1, 2020

Published: September 4, 2020

Copyright (อ 2020 by author(s) and Scientific Research Publishing Inc. This work is licensed under the Creative Commons Attribution International License (CC BY 4.0).

http://creativecommons.org/licenses/by/4.0/ (c) (i) Open Access

\begin{abstract}
Geometric properties of trajectories of angled projectiles under gravity pull are a popular common traditional theme discussed in introductory physics and engineering college courses. What is overlooked is the universal collective properties of the overarching specificities of families of such parabolas, the envelope. For instance [1] and references within explored the existence of one such envelope, however, even the most recent article [2] overlooked its global hidden properties. Here, we investigate exposing this hidden information. Having the equation of the envelope on hand we introduce its universal characteristics such as its: arc length, enclosed 2D surface area, surface area of the surface-of-revolution about the symmetry axis, and, the volume of the enclosure. Numeric values of these quantities are global as is e.g. the $45^{\circ}$ projectile angle that maximizes the range of a projectile in vacuum irrespective, its initial speed. In our exploratory investigation, we utilize the popular Computer Algebra System (CAS) Mathematica ${ }^{\mathrm{TM}}$ [3] [4] [5].
\end{abstract}

\section{Keywords}

Family of Angled Projectiles, Envelope, Mathematica

\section{Motivations and Goals}

The absentees of physical media such as air suppress the impact of viscosity, velocity-dependent forces, size and spin of projectiles and their cohesive combined effects, i.e. the Magnus effect, when analyzing the features of projectile motion. The impact of the individual elements and the Magnus effect are discussed in detail by the author [6] and to some extent in references within. Ignoring the Magnus effect and all the aforementioned elements makes the gravity the sole agent acting on a projectile projected in a vertical plane. Consequently, this formulates the classic projectile problem. The geometric properties and associated 
physics issues of a single point-like object thrown above the horizontal are discussed even in introductory physics and engineering texts [7]. Noticing, the common theme of all these studies with no exception hovers always about a single projectile and its associated single trajectory.

A few articles have gone beyond the scope of the aforementioned classic limits exploring the collective properties of family of trajectories, i.e. the envelope of the trajectories e.g. [1], 2003. Because of the continued interest, similar articles have been published, e.g. a recent [2], 2019. Reviewing these articles and references within reveals their common theme, derivation of the envelope equation. Nonetheless none has gone beyond the classic scope.

With this observation, we establish the motivation for our exploratory research by taking the known information as a background adding new material augmenting the classic scope. In analysis section, we present a short review of the current status of information concerning the envelope of a family of trajectories of projectiles in a vertical plane under the sole influence of gravity. This section includes detailed calculation conducive to a set of fresh information. This reveals the overlooked properties of the envelope. For instance, it gives among other things the arc-length of the envelope, the surface area of the surface-of-revolution of the envelope. These are useful fresh information as is the known classic $45^{\circ}$ projectile angle that maximizes the Range of a projectile.

To achieve these goals, calculations are performed analytically along with adapting the symbolic and numeric features of CAS, Mathematica V12.1, specifically. Graphs are made with the aforementioned software.

This article is comprised of three sections. Section 1 is the Motivations and Goals; Section 2 is the Analysis; Section 3 is the Summary and Conclusions.

\section{Analysis}

In a vertical plane with the upright direction being the $+y$-axis with the left-to-right direction $+x$-axis time-dependent coordinate of a point on the trajectory of a projectile thrown at angle, $\theta$, above the horizontal with initial speed, $v_{0}$, is [7],

$$
\{x(t), y(t)\}=\left\{v_{0} \cos (\theta) t,-\frac{1}{2} g t^{2}+v_{0} \sin (\theta) t\right\}
$$

where $g$ is the gravity acceleration. By eliminating time, $t$, between $x(t)$ and $y(t)$, Equation (1) yields,

$$
y(x, \theta)=-\frac{1}{2} \frac{g}{v_{0}^{2} \cos ^{2}(\theta)} x^{2}+\tan (\theta) x
$$

Since the projectiles are considered to have the same initial speeds and gravity acts evenly on all, the problem poses itself as a pure geometry problem. Accordingly, without losing the generalities we set the initial speeds and the gravity acceleration to unity. Equation (2) becomes,

$$
y(x, \theta)=-\frac{1}{2 \cos ^{2}(\theta)} x^{2}+\tan (\theta) x
$$


For a chosen initial angle, $\theta$, Equation (3) plots a classic parabolic trajectory. By varying, $\theta$, the $\theta$-dependent parametric Equation (3) plots a family of such curves shown in Figure 1. The symmetry of the trajectories about the main vertical axis, $y$-axis, by replacing $\theta \rightarrow \pi-\theta$ is shown as well. The latter curves are the mirror images of the ones shown in $x>0$ region as if the projectiles were projected in the $x<0$ zone.

By inspecting curves shown in Figure 1, one realizes the larger the projectile angle the higher is the trajectory corresponding to a shorter abscissa. This figure shows also what the envelope is; it is a curve that embodies all the parabolas touching individual trajectory at one point only. Simply put the envelope is a unique curve that is tangent to the family of curves. One attempts almost to run a fingertip on Figure 1 outlining the envelope. Such an outlined curve would almost trace a parabola. To identify the curve, we follow the standard procedure. We set the slope of Equation (3) with respect to, $\theta$, zero. Then we eliminate the $\theta$ between the Equation (3) and the latter. This procedure is cumbersome and tedious. Instead, we reformat Equation (3) and introduce a new variable, $\xi=$ $\tan (\theta)$. In terms of, $\xi$, Equation (3) is,

$$
y(x, \xi)=-\frac{1}{2}\left(1+\xi^{2}\right) x^{2}+\xi x
$$

the needed slope is, $\frac{\mathrm{d}}{\mathrm{d} \theta} y(x, \theta)$. In terms of, $\xi$ it is $\frac{\mathrm{d}}{\mathrm{d} \theta} y(x, \theta)=\frac{\mathrm{d} \xi}{\mathrm{d} \theta} \frac{\mathrm{d}}{\mathrm{d} \xi} y(x, \xi)$, where $\frac{\mathrm{d} \xi}{\mathrm{d} \theta}=1+\xi^{2}$. These yields,

$$
\frac{\mathrm{d}}{\mathrm{d} \theta} y(x, \theta)=x\left(1+\xi^{2}\right)(1-\xi x)
$$

Setting Equation (5) $=0$ gives, $\xi=1 / x$. Substituting in Equation (4) yields the envelope equation,

$$
y(x)=\frac{1}{2}\left(1-x^{2}\right)
$$

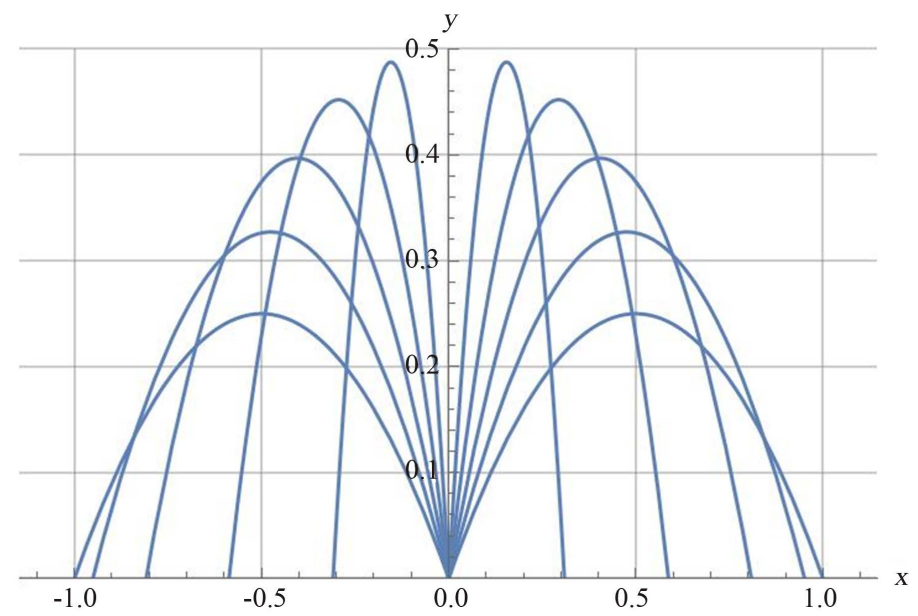

Figure 1. Plots of classic parabolic trajectories. 
Sketch of Equation (6), the envelope, along with Figure 1 is shown in Figure 2.

Trajectories commonly are plotted in the upper half-plane as shown in Figure 2, we display the envelope in the same plane as well. These are the pictorial known classic information about the envelope. We are augmenting this information six-fold as follows:

1) Shown in Figure 2, the maximum height of the envelope occurs at $x=0$, corresponding to $y_{\max }=1 / 2$. This conforms utilizing, $y^{\prime}(x)=0$.

2) Equation (6) intersects the $x$-axis at two points, $x= \pm 1$. Although the envelope does not represent a trajectory of a projectile, but one may think of a scenario that it does. Meaning, a projectile projected from either one of these two points to the opposite direction travels horizontally to the maximum Range. The slopes of the envelope at these points are \pm 1 corresponding to $135^{\circ}$ and $45^{\circ}$, respectively. These are the same classic angles that maximize the Range of a projectile; recall Range $=\sin (2 \theta)$, with $R_{\max }=1$, corresponding to $\theta=45^{\circ}$, [7] .

3) The arc-length of the envelope. This is subject to, $\ell=\int \sqrt{1+y^{\prime}(x)^{2}} \mathrm{~d} x$. Utilizing Equation (6) gives,

$$
\ell=\int_{-1}^{1} \sqrt{1+x^{2}} \mathrm{~d} x=\sqrt{2}+\operatorname{Arcsinh}(1)
$$

Its numeric value is, 2.29. This is a global numeric value; it is independent of the values of initial speed, gravity acceleration and the initial projectile angle. It is a generic quantity associated with the envelope of the classic projectile problem.

4) The surface area of the enclosed surface. This is subject to, $s=\int y(x) \mathrm{d} x$. Utilizing Equation (6) gives,

$$
S=\int_{-1}^{1} \frac{1}{2}\left(1-x^{2}\right) \mathrm{d} x=\frac{2}{3}
$$

5) Surface area of paraboloid. By rotating the envelope which is a parabola about its vertical symmetry axis a paraboloid is formed. This is shown on the right panel of Figure 3.

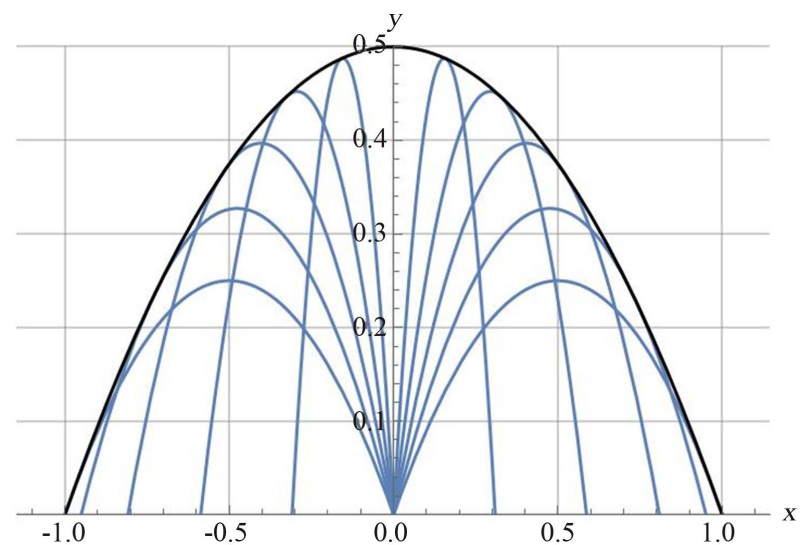

Figure 2. The black curve is the envelope, the families of the parabolic trajectories are in blue. 

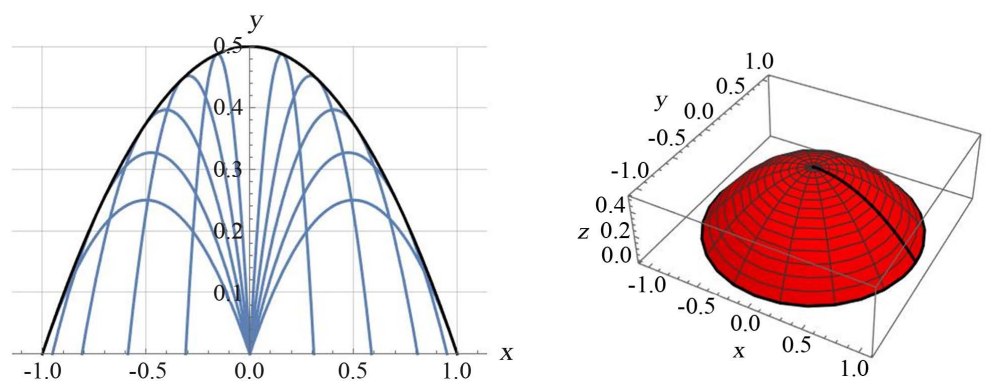

Figure 3. The right panel is the display of the paraboloid that is generated by rotating the envelope about the $y$-axis. The left panel is the same as Figure 2.

The surface area of the paraboloid is subject to, $S=2 \pi \int x \sqrt{1+y^{\prime}(x)^{2}} \mathrm{~d} x$. Utilizing Equation (6) gives,

$$
S=2 \pi \int_{0}^{1} x \sqrt{1+x^{2}} \mathrm{~d} x
$$

This is, $2 / 3(2 \sqrt{2}-1) \pi$, it evaluates, 3.83 . The surface area of the paraboloid is another global quantity associated with the parabolic envelope.

And finally,

6) The volume of the paraboloid. The right panel of Figure 3 shows the $3 \mathrm{D}$ surface of the surface-of-revolution of the envelope, this surface encloses a volume. Its value is subject to, $V=2 \pi \int x y(x) \mathrm{d} x$. Utilizing Equation (6), gives,

$$
V=\pi \int_{0}^{1} x\left(1-x^{2}\right) \mathrm{d} x
$$

Evaluates, $\pi / 4$.

\section{Discussion and Conclusions}

In this investigating research project, we revisited the classic projectile problem in the absentees of any media other than the mere gravitational pull. Briefly, we reviewed the essentials of the projectile motion, its kinematics, conducive to derivation of the envelope equation. Having established this basis, we augmented the classic scope of the characteristics of the envelope merely from geometric point of view. Exhaustive literature search reveals the nonexistence point of view discussed in our investigation. Although calculation conducive to our finding is straight forward and trivial, presented results are as interesting as some of the classic known features associated with the projectile motion. Our investigation reveals the hidden unreported geometric properties of the envelope. For instance, we calculate the arc-length of the envelope, the surface area enclosed by the envelope, the surface area of the surface-of-revolution among other things. A short summary of our investigation is tabulated below.

\begin{tabular}{cc}
\hline Arclength of Envelope & $\sqrt{2}+\operatorname{Arcsinh}(1)=2.29$ \\
Surface Area of Envelope & $2 / 3$ \\
Surface Area of Paraboloid & $2 / 3(2 \sqrt{2}-1) \pi=3.83$ \\
Volume of Paraboloid & $\pi / 4$ \\
\hline
\end{tabular}




\section{Acknowledgements}

The author acknowledges the support of the John T. and Paige S. Smith professorship funds completing this work.

\section{Conflicts of Interest}

The author declares no conflicts of interest regarding the publication of this paper.

\section{References}

[1] Butikov, E. (2003) Comment on the Envelope of Projectile Trajectories. European Journal of Physics, 24, L5-L9.

https://iopscience.iop.org/article/10.1088/0143-0807/24/4/101/meta https://doi.org/10.1088/0143-0807/24/4/101

[2] Mihas, P. (2019) Envelopes, Foci, and Maxima of Special Trajectories. American Journal of Physics, 87, 836. https://doi.org/10.1119/1.5122894

[3] http://www.wolfram.com/

[4] Wolfram, S. (1996) Mathematica Book. 3rd Edition, Cambridge University Press, Cambridge.

[5] Sarafian, H. (2019) Mathematica Graphics Examples. 2nd Edition, Scientific Research Publishing.

[6] Sarafian, H. (2015) Impact of the Drag Force and the Magnus Effect on the Trajectory of a Baseball. World Journal of Mechanics, 5, 49-58. https://doi.org/10.4236/wjm.2015.54006

[7] Halliday, D., Resnick, R. and Walker, J. (2013) Fundamentals of Physics Extended. 10th Ed, John Wiley and Sons, NY. 